\title{
JPN Guidelines for the management of acute pancreatitis: severity assessment of acute pancreatitis
}

\author{
Masahiko Hirota ${ }^{1}$, Tadahiro Takada 2 ,*, Yoshifumi Kawarada 3 , Koichi Hirata 4 , Toshihiko Mayumi 5 , \\ Masahiro Yoshida ${ }^{2}$, Mino Sekimoto ${ }^{6}$, Yasutoshi Kimura ${ }^{4}$, Kazunori Takeda ${ }^{7}$, Shuji Isaji ${ }^{8}$, Masaru Koizumi ${ }^{9}$, \\ Makoto Otsuki ${ }^{10 * * *}$, and Seiki Matsuno ${ }^{11, * * *}$ \\ ${ }^{1}$ Department of Gastroenterological Surgery, Kumamoto University Graduate School of Medical Sciences, 1-1-1 Honjo, \\ Kumamoto 860-0811, Japan \\ ${ }^{2}$ Department of Surgery, Teikyo University School of Medicine, Tokyo, Japan \\ ${ }^{3}$ Ueno Municipal Hospital, Mie, Japan \\ ${ }^{4}$ First Department of Surgery, Sapporo Medical University School of Medicine, Hokkaido, Japan \\ ${ }^{5}$ Department of Emergency and Critical Care Medicine, Nagoya University Graduate School of Medicine, Nagoya, Japan \\ ${ }^{6}$ Department of Healthcare Economics and Quality Management, Kyoto University Graduate School of Medicine, Kyoto, Japan \\ ${ }^{7}$ Department of Surgery, National Hospital Organization Sendai Medical Center, Sendai, Japan \\ ${ }^{8}$ Department of Hepatobiliary Pancreatic Surgery and Breast Surgery, Mie University Graduate School of Medicine, Mie, Japan \\ ${ }^{9}$ Ohara Medical Center Hospital, Fukushima, Japan \\ ${ }^{10}$ Department of Gastroenterology and Metabolism, University of Occupational and Environmental Health, Japan, School of Medicine, \\ Kitakyushu, Japan \\ ${ }^{11}$ Division of Gastroenterological Surgery, Tohoku University Graduate School of Medicine, Sendai, Japan
}

\begin{abstract}
This article addresses the criteria for severity assessment and the severity scoring system of the Ministry of Health and Welfare of Japan; now the Japanese Ministry of Health, Labour, and Welfare (the JPN score). It also presents data comparing the JPN score with the Acute Physiology and Chronic Health Evaluation (APACHE) II score and the Ranson score, which are the major measuring scales used in the United States and Europe. The goal of investigating these scoring systems is the achievement of earlier diagnosis and more appropriate and successful treatment of severe or moderate acute pancreatitis, which has a high mortality rate. This article makes the following recommendations in terms of assessing the severity of acute pancreatitis:
\end{abstract}

(1) Severity assessment is indispensable to the selection of proper initial treatment in the management of acute pancreatitis (Recommendation A).

(2) Assessment by a severity scoring system (JPN score, APACHE II score) is important for determining treatment policy and identifying the need for transfer to a specialist unit (Recommendation A).

(3) C-reactive protein (CRP) is a useful indicator for assessing severity (Recommendation A).

Offprint requests to: $\mathrm{M}$. Hirota

* President, Japanese Society of Emergency Abdominal Medicine; President, Japanese Society of HepatoBiliary-Pancreatic Surgery; President; Asian-Pacific Hepato-Pancreato-Biliary Association

** Chairman, Intractable Pancreatic Disease Investigation and Research Group of the Japanese Ministry of Health, Labour, and Welfare

*** President, Japan Pancreas Society
(4) Contrast-enhanced computed tomography (CT) scanning and contrast-enhanced magnetic resonance imaging (MRI) play an important role in severity assessment (Recommendation A).

(5) A JPN score of 2 or more (severe acute pancreatitis) has been established as the criterion for hospital transfer (Recommendation A).

(6) It is preferable to transfer patients with severe acute pancreatitis to a specialist medical institution where they can receive continuous monitoring and systemic management.

Key words Severity assessment $\cdot$ Acute pancreatitis $\cdot$ JPN score $\cdot$ APACHE II score

\section{Clinical questions}

CQ1. Is severity assessment necessary in the management of acute pancreatitis?

CQ2. Are severity scoring systems (JPN score, Acute Physiology and Chronic Health Evaluation [APACHE] II score) useful for assessing the severity of acute pancreatitis?

CQ3. Are clinical symptoms and signs useful for severity assessment of acute pancreatitis?

CQ4. Are blood tests useful for severity assessment of acute pancreatitis?

CQ5. Is diagnostic imaging useful for severity assessment of acute pancreatitis?

CQ6. What are the indications for transferring patients with acute pancreatitis to a specialist unit? 


\section{Introduction}

There is wide variation in the severity of acute pancreatitis. Mild acute pancreatitis tends towards spontaneous remission, but once acute pancreatitis becomes severe, there is a great risk of death from fatal complications, such as circulatory failure, vital organ failure, and infection. Because acute pancreatitis, although benign, has a poor prognosis, it has been designated as an intractable disease by the Japanese Ministry of Health, Labour, and Welfare. To improve prognosis, it is important to assess accurately the severity of the disease in order to select proper initial treatment. Thus, accurate severity assessment and determination of a proper disease management policy are crucial. The severity scoring system most widely used in Japan (JPN score) allows sequential scoring of severity and is useful for the selection of proper initial treatment. The JPN scoring system is based on clinical signs, blood test data, and imaging findings, and it is used to decide on the treatment strategy. Because acute pancreatitis that is initially diagnosed as mild or moderate can progress to severe, or become fatal, during treatment, even mild acute pancreatitis should be continuously monitored with the greatest care. Serial assessments of severity should be performed as early as possible to detect signs of a poor outcome. This article reviews the JPN score, various severity evaluation systems used in the United States and Europe, and severity assessment methods that are based on clinical signs and blood tests. Practical recommendations were also graded according to suggestions in previous reports. ${ }^{1,2}$

\section{Necessity for severity assessment}

Clinical question (CQ) 1. Is severity assessment necessary in the management of acute pancreatitis?

Severity assessment is essential to the selection of appropriate initial treatment. Even acute pancreatitis that is initially diagnosed as mild or moderate may quickly progress to severe, and continuous assessment is required, particularly during the first 3 days after onset (Recommendation A)

To improve the survival rate in patients with acute pancreatitis, severity assessment during the initial examination is extremely important to ensure the quick and accurate diagnosis of severe cases (which have a high mortality rate), to commence appropriate initial treatment, and, if necessary, to transfer the patient to an advanced specialist medical institution. Because quite a few cases initially diagnosed as mild acute pancreatitis may progress to severe acute pancreatitis, serial assessments of severity should be performed. It is also impor- tant to monitor the effects of treatment by serial assessments of severity.

When deciding on an appropriate system of criteria to assess the severity of pancreatitis, it is important to establish whether the assessment (1) depends on an examination method that is available at most medical institutions, (2) can be used to assess severity shortly after admission to the hospital, and (3) can be used to monitor the course of the disease, and can be preformed repeatedly.

\section{Severity scores}

CQ2. Are severity scoring systems (JPN score, Acute Physiology and Chronic Health Evaluation [APACHE] II score) useful for assessing the severity of acute pancreatitis?

Assessment by a severity scoring system is important when deciding on treatment policy and the need for transfer to a specialist unit. Use of the JPN score is recommended for severity assessment in Japan (Recommendation A)

\section{Historical progress}

Because acute pancreatitis is manifested by a wide range of clinical signs, it is difficult to evaluate those signs objectively as parameters for severity assessment. With this feature in mind, severity assessment criteria, in the form of the Ranson score, were proposed in 1974 in the United States. Of 43 total criteria, Ranson et al. ${ }^{3}$ selected those 11 that showed statistical significance, consisting mainly of examination findings (Level 1b). ${ }^{3}$ The criteria were not suitable for patients with gallstone pancreatitis and so were evaluated only for patient groups containing a higher percentage of patients with alcoholic pancreatitis; subsequently, in 1982, new separate assessment criteria, classified according to gallstone, alcoholic, and other types of pancreatitis, were prepared (the Ranson score will be detailed later). ${ }^{4}$ In 1978, 9 severity assessment criteria, comprised mainly of examination findings, were developed in the United Kingdom (Level 1b). ${ }^{5}$ After two revisions,${ }^{6,7}$ these criteria are currently being used as the Glasgow score. These two sets of severity assessment criteria (i.e., the revised Rarson score ${ }^{4}$ and the Glasgow score ${ }^{6,7}$ ) are used widely throughout the world. The scoring system of Bank et al. ${ }^{8}$ which uses clinical symptoms as important criteria, is also used. In 1964, Forell, ${ }^{9}$ in Germany, reported clinical and examination items that he considered indicative of severe pancreatitis, and Damman et al..$^{10}$ reported assessment criteria consisting of eight indicators in 1981.

In Japan, assessment criteria consisting of symptoms and blood tests and computed tomography (CT) scan 
Table 1. Standardized criteria for severity grading of acute pancreatitis

A. Standardized criteria for grading the severity of acute pancreatitis

\begin{tabular}{|c|c|c|}
\hline Factor & Clinical signs & Laboratory data \\
\hline $\begin{array}{l}\text { Prognostic factor I ( } 2 \text { points for } \\
\text { each positive factor) }\end{array}$ & $\begin{array}{l}\text { - Shock } \\
\text { - Respiratory failure } \\
\text { - Mental disturbance } \\
\text { - Severe infection } \\
\text { - Hemorrhagic diathesis }\end{array}$ & $\begin{array}{l}\text { - } \mathrm{BE} \leqq-3 \mathrm{mEq} / \mathrm{l} \\
\text { - } \mathrm{Ht} \leqq 30 \% \text { (after hydration) } \\
\text { - } \mathrm{BUN} \geqq 40 \mathrm{mg} / \mathrm{dl} \text { or creatinine } \geqq 2.0 \mathrm{mg} / \mathrm{dl}\end{array}$ \\
\hline $\begin{array}{l}\text { Prognostic factor II ( } 1 \text { point for } \\
\text { each positive factor) }\end{array}$ & & $\begin{array}{l}\text { - } \mathrm{Ca} \leqq 7.5 \mathrm{mg} / \mathrm{dl} \\
\text { - } \mathrm{FBS} \geqq 200 \mathrm{mg} / \mathrm{dl} \\
\text { - } \mathrm{PaO}_{2} \leqq 60 \mathrm{mmHg} \text { (room air) } \\
\text { - } \mathrm{LDH} \geqq 700 \mathrm{IU} / \mathrm{l} \\
\text { - } \text { Total protein } \leqq 6.0 \mathrm{~g} / \mathrm{dl} \\
\text { - } \text { Prothrombin time } \geqq 15 \mathrm{~s} \\
\text { - } \text { Platelet count } \leqq 1 \times 10^{5} / \mathrm{mm}^{3} \\
\text { - } \mathrm{CT} \mathrm{Grade} \mathrm{IV} \mathrm{or} \mathrm{V}^{\mathrm{a}}\end{array}$ \\
\hline
\end{tabular}

Prognostic factor III

- SIRS score $\geqq 3$ (2 points)

- Age $\geqq 70$ years (1 point)

B. Stage classification of acute pancreatitis

Stage 0 , mild acute pancreatitis

Stage 1, moderate acute pancreatitis

Stage 2, severe acute pancreatitis I (severity score, $2-8$ points)

Stage 3, severe acute pancreatitis II (severity score, 9-14 points)

Stage 4, extremely severe acute pancreatitis (severity score, 15-27 points)

Standardized criteria

Severe, If at least one item in prognostic factor I is present, or if more than two items in prognostic factor II are present, the case is considered severe. Moderate, if none of the items in prognostic factor I and only one item in prognostic factor II is present, the case is considered moderate. Mild, if none of the items in prognostic factor I or II are present, the case is considered mild

Severity score

The sum of the points for the positive prognostic factors is defined as the severity score

Data are from reference 9

${ }^{a}$ If diffuse, uneven density is present in the pancreatic parenchyma, or if extrapancreatic inflammatory changes extend away from the pancreas, the case is considered as Grade IV or Grade V on CT

findings were developed in 1990 by the then Research Committee for Intractable Diseases of the Pancreas, which was sponsored by the then Japanese Ministry of Health and Welfare, and these criteria have been applied clinically. In 1998, the severity scoring system (JPN score) was developed and the 2002 version $^{11}$ is summarized in Table 1.

For a long time, various severity assessment criteria were based on the analysis of clinical data, but they have now been modified to take into account the etiology, the age and sex of the patient, examination and treatment methods, and all other applicable factors.

In 1981, the APACHE score was developed as a method for assessing the severity of acute diseases from the standpoint of emergency treatment. The APACHE II score (see below), renamed after modification in $1985,{ }^{12}$ enjoys a good reputation as a tool for assessing the severity of acute pancreatitis.

\section{Evaluation of severity scores}

Ranson score and Glasgow score

The Ranson and Glasgow scoring systems are based mainly on examination findings and have a sensitivity of between $70 \%$ and $80 \%$ for predicting a poor outcome (Levels 1b-2b). ${ }^{3,4,7,13,14}$ Metaanalysis (prediction of a poor outcome) suggested that the Ranson and Glasgow scores were almost equal as assessment criteria, but neither of them were satisfactory (Level 1a). ${ }^{14,15}$ Both require $48 \mathrm{~h}$ to complete assessments.

\section{APACHE II score}

The APACHE II score is based on 12-item findings in the medical examination, plus the presence of chronic complications and age; it is considered to be a useful tool for assessing severity in $24 \mathrm{~h}$ (Levels $2 \mathrm{a}-2 \mathrm{~b}) .{ }^{16,17}$ Daily scoring enables monitoring of the course of pancreatitis. The Atlanta Symposium in $1992^{18}$ concluded that a score of 8 or more indicated severe pancreatitis, while the Santorini Consensus Conference, in 1999, ${ }^{19}$ concluded that a score of 6 or more indicated severe pancreatitis; both gatherings rated the APACHE II score as the best assessment tool, because the results can be obtained so quickly. The British Society of Gastroenterology $(1998)^{20}$ suggests that the APACHE II score is useful for early diagnosis and monitoring of the clinical course, citing a report (Level $2 \mathrm{a})^{17}$ stating that 
when an APACHE II score of 6 or more is used as a criterion for severe disease, the positive predictive value is $40 \%$ (sensitivity, $95 \%$ ).

JPN score (Table 1) $)^{11}$

The criteria for the JPN score were developed based on an analysis of the results of nationwide surveillance. Five clinical sign items, ten blood test items, CT findings, the presence of systemic inflammatory response syndrome (SIRS), and age were all scored as prognostic factors. The highest possible total score is 27, and severity is classified into five stages (Stages 0 to 4 ). ${ }^{11}$ Examination of about 1100 patients showed that the scores were correlated with outcome (Level 3b). ${ }^{21}$ Examination of the results of a nationwide survey of 1240 patients conducted between 1995 and 1998 showed that the JPN score had almost the same value for assessment as the APACHE II score and the Ranson score (Level $3 b) .{ }^{22}$

However, because the currently used severity assessment criteria involve too many evaluation items and it is difficult to interpret data obtained during oxygen administration or transfusion, new severity assessment criteria, based on nine evaluation items, are now being developed.

\section{Clinical symptoms and signs}

CQ3. Are clinical symptoms and signs useful for severity assessment of acute pancreatitis?

Because acute pancreatitis is manifested by a wide range of clinical signs, it is difficult to evaluate them objectively as parameters for use in severity assessment.

Patients exhibiting manifestations of vital organ failure, such as shock, psychoneurotic symptoms, and abdominal distention (ileus, ascites), are assessed as having severe acute pancreatits, and these manifestations have been used as parameters for reported severity assessment criteria. ${ }^{5,89}$ Five conditions - shock, dyspnea, neurotic symptoms, severe infection, and bleeding tendency (including that manifested by Grey-Turner's sign and Cullen's sign) - are considered to be associated with a prognosis for survival. ${ }^{11}$ Analysis of different severity assessment criteria has revealed that criteria based on clinical findings have a sensitivity of $54.7 \%$, a specificity of $93.0 \%$, and a positive predictive value of $59.2 \%$, while criteria based on examination findings have a sensitivity of $69.3 \%$, a specificity of $79.5 \%$, and a positive predictive value of $77.1 \%$ (Level 1a). ${ }^{15}$ This suggests that clinical findings alone are not reliable for severity assessment, and that further investigation is required, because both clinical and examination findings have advantages and disadvantages.
Abdominal findings in the form of color marks on the skin, e.g., Grey-Turner's sign, Cullen's sign, and Fox's sign, are indicative of a poor outcome and high mortality, ${ }^{23,24}$ but they do not necessarily reflect severity. ${ }^{25}$ The value of the color marks for severity assessment remains obscure, and, because they often appear 48 to $72 \mathrm{~h}$ after the onset of pancreatitis, their significance as an early predictor of poor outcome is low.

The 1992 Atlanta Symposium, ${ }^{18}$ an international conference that examined severity assessment of acute pancreatitis, suggested that clinical organ failure (respiration, circulatory, and kidney failure) was an indicator of severe pancreatitis, while the guidelines of the British Society of Gastroenterology ${ }^{20}$ state that clinical assessment alone is unreliable and will misclassify around $50 \%$ of patients (Level 2b). ${ }^{26}$ The Santorini Consensus Conference in $1999^{19}$ suggested that skin findings such as Grey-Turner's sign and Cullen's sign were signs of severity.

\section{Severity assessment based on blood tests}

CQ4. Are blood tests useful for severity assessment of acute pancreatitis?

Serum C-reactive protein (CRP) values

Serum CRP values are useful for severity assessment, but they may not reflect severity within the first $48 \mathrm{~h}$ after onset (Recommendation A)

Serum CRP values are a sensitive predictor of the progression of severity from moderate to severe. The time of measurement and the CRP values vary with different investigations and researchers. In one report, the cutoff value within the first $48 \mathrm{~h}$ of onset was greater than $30 \mathrm{mg} / \mathrm{dl}$ (Level 1c), ${ }^{27}$ whereas it was greater than $15 \mathrm{mg} / \mathrm{dl}$ in other reports (Level 2b). ${ }^{28,29}$ The Santorini Consensus Conference in $1999^{19}$ recommended a cutoff value of greater than $15 \mathrm{mg} / \mathrm{dl}$. There is one report that shows the peak CRP in patients with severe cases at more than ten times above normal (less than five times above normal in mild cases; $P<0.001$; Level 2b), ${ }^{30}$ and another report has suggested that peak CRP greater than $21 \mathrm{mg} / \mathrm{dl}$ within the first 4 days after onset (greater than $12 \mathrm{mg} / \mathrm{dl}$ after 7 days) can be used as a predictor of progression to severity, with a sensitivity of about $80 \%$ (Level 2b). ${ }^{31}$ Still another report (Level 1c) ${ }^{27}$ shows that combining CRP with other diagnostic criteria further improves sensitivity. However, it should be noted that the CRP values may not reflect severity before $48 \mathrm{~h}$ after onset.

There is a report (Level $2 b)^{32}$ stating that CRP values are correlated with the CT severity index (Level 1c) ${ }^{33}$ when the maximum CRP (within $72 \mathrm{~h}$ of onset) is greater than ten times the normal value. 
CRP values are of little use as a diagnostic indication for infected pancreatic necrosis, but if CRP measured on admission is greater than $8.5 \mathrm{mg} / \mathrm{dl}$, sepsis can be diagnosed with a sensitivity of $100 \%$, a specificity of $53 \%$, a positive predictive value of $50 \%$, and a true positive rate of $68 \%$ (Level $1 \mathrm{~b}$ )..$^{31}$ This suggests that CT should be used for the diagnosis of infected pancreatic necrosis (true positive rate, $81 \%$ ).

\section{Hematocrit (Hct)}

Hemoconcentration caused by dehydration is a predictor of pancreatic necrosis and organ failure (Level 2b). ${ }^{34,35}$ If the Hct on admission is $47 \%$ or more, or if no improvement is observed within the first $24 \mathrm{~h}$, there is a strong possibility that pancreatic necrosis has begun (sensitivity, $81 \%$; specificity, $88 \%$ ). ${ }^{34}$

\section{Serum phospholipase $A_{2}\left(P L A_{2}\right)$}

The serum PLA $_{2}$ level in severe pancreatitis is already significantly higher on the day of onset than it is in moderate pancreatitis, and the degree of elevation is much greater than the degree of amylase elevation or the degree of trypsin elevation (Level 2b). ${ }^{36}$ Type IB secretory $\mathrm{PLA}_{2}\left(\mathrm{sPLA}_{2}\right)$ (a digestive enzyme present in pancreatic juice) and type IIA sPLA $_{2}$ (produced by various cells in response to inflammatory stimuli) are both low-molecular-weight PLA , while cytosolic PLA $_{2}$ $\left(\mathrm{cPLA}_{2}\right)$ (contained in the cytoplasm and involved in arachidonic acid metabolism) is a high-molecularweight $\mathrm{PLA}_{2}$; all have been identified as isoenzymes.

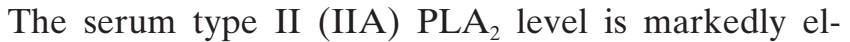
evated in patients with severe acute pancreatitis (Level 3b). ${ }^{37}$ SIRS-positive patients have significantly higher $\mathrm{PLA}_{2}$ levels (Level 2b), ${ }^{38}$ and the PLA ${ }_{2}$ level is even higher in patients with more SIRS-positive items (Level 2b). ${ }^{38}$ Increases in the $\mathrm{PLA}_{2}$ level are considered to be evidence of systemic inflammation leading to multiple organ failure. However, no significant association between serum $\mathrm{PLA}_{2}$ values and severity or mortality has been demonstrated.

\section{Diagnostic imaging}

CQ5. Is diagnostic imaging useful for severity assessment of acute pancreatitis?

The presence and range of pancreatic necrosis and the extent of inflammatory change are correlated with severity. Accurate diagnosis of the presence and range of pancreatic necrosis requires contrast-enhanced $\boldsymbol{C T}$ or contrast-enhanced magnetic resonance imaging (MRI). However, it should be noted that the use of contrast medium may involve adverse reactions (Recommendation A)

\section{Computed tomography $(C T)$}

It is important to diagnose accurately the presence of pancreatic necrosis and the extent of inflammatory change, both of which are closely associated with various complications and prognosis (Levels 1b-3b) $29,39,40$ and affect treatment policy, such as the administration of an antibacterial agent to prevent infection. Plain CT scans allow for an evaluation of peripancreatic inflammatory change, but they can not be used to diagnose pancreatic necrosis in general. Contrast-enhanced $\mathrm{CT}$ is required for the accurate diagnosis of the presence and range of pancreatic necrosis (Level 1c). ${ }^{41}$ One report (Level 2b) ${ }^{42}$ has shown that the use of a contrast medium does not aggravate the morbidity of pancreatitis, while another (Level $2 \mathrm{~b})^{43}$ argues that it exacerbates pancreatitis. As the contrast medium may probably aggravate kidney disorders associated with severe acute pancreatitis, contrast-enhanced CT should be used only when the value of the information obtained exceeds the disadvantages, such as impairment of renal function and allergic reactions. Contrast-enhanced CT has various other advantages besides the visualization of pancreatic necrosis. It provides useful information for evaluating the need for surgical procedures and drainage, and it visualizes pseudoaneurysms. There is also an opinion that contrast-enhanced CT should only be used when a case has been diagnosed as severe by plain CT (Level 2b). ${ }^{44}$

The CT severity index (Level 2b), ${ }^{33}$ which is based on CT findings, is also used for severity assessment. It scores severity based on a combination of the presence and extent of pancreatic necrosis and the extent of peripancreatic inflammatory change, all of which are closely associated with outcome. A modified CT severity index (Level 2b), ${ }^{45}$ which simplifies the evaluation of pancreatic necrosis and inflammatory changes in and around the pancreas and evaluates the presence of extrapancreatic complications, has also been proposed. In Japan, Matsuno et al. ${ }^{46}$ proposed a severity assessment method, using contrast-enhanced CT, from a similar standpoint, and they also reported on its usefulness (Level 2b).

It is desirable to use contrast-enhanced $\mathrm{CT}$ in patients with a high JPN score, APACHE II score, and Ranson score (see below), and in those patients with organ failure. Contrast-enhanced CT is the most useful method for differentiating edematous from necrotizing pancreatitis (Level 1c). ${ }^{41}$

A small unenhanced area on contrast-enhanced CT scans indicates edematous change in the pancreatic parenchyma. A large unenhanced area indicates pancreatic necrosis. At the Atlanta Symposium in 1992,18 poorly perfused area (having an increase in density after enhancement of less than 30 Hounsfield units 
[Level 1c] $]^{41}$ ) covering more than $30 \%$ of the pancreas or measuring $3 \mathrm{~cm}$ or more in diameter was defined as diagnostic evidence of pancreatic necrosis. However, recently, smaller unenhanced areas are often being evaluated as a sign of pancreatic necrosis. There is no clear criterion for distinguishing edematous changes from pancreatic necrosis in a small area.

The Guidelines of the British Society of Gastroenterology ${ }^{20}$ recommend that dynamic contrast-enhanced CT in patients with severe acute pancreatitis should be performed between the third and tenth days of hospital admission. This is based on the findings that necrosis is often not evident in the initial stage and that it is better to perform CT scanning after initial treatment. Contrast-enhanced CT between the fourth and tenth days after onset enables diagnosis of pancreatic necrosis to almost 100\% accuracy (Level 1b-2b). ${ }^{29,39,41,47}$ However, some studies in Europe have reported that contrastenhanced CT on admission (within the first 36 to $48 \mathrm{~h}$ ) is useful for assessing the severity of acute pancreatitis (Level 2b). ${ }^{48,49} \mathrm{CT}$ is often performed on the day of admission in order to determine whether there is an indication for continuous regional arterial infusion therapy. Even after the initial examination, CT scanning needs to be applied regularly and whenever any infection or other complications are suspected. The best time to perform CT should be determined by further investigations, so as to resolve the differences in opinion between researchers in Japan, the United States, and Europe.

\section{Magnetic resonance imaging (MRI)}

Contrast-enhanced MRI can be used to diagnose the presence of pancreatic necrosis and inflammatory changes in and around the pancreas, and it is also useful for assessing severity (Level 2b). ${ }^{50-53}$ MRI has the advantages of (1) less nephrotoxicity of the contrast medium, (2) no X-ray exposure, (3) allowing evaluation of damage to the pancreatic duct, and (4) providing information on the biliary system. MRI is useful for identifying the etiology of acute pancreatitis and is not merely an alternative procedure for use in patients who cannot undergo CT scanning. However, MRI also has disadvantages. For example: (1) because metal objects cannot be brought into the scanning area, MRI cannot be used in patients who are on a respirator; (2) timeconsuming procedures, including the removal of transfusion pumps, must be completed before MRI can be performed; and (3) no emergency MRI system has been properly established.

\section{Plain X-rays of the chest and abdomen}

It has been reported that early pleural effusion in patients with acute pancreatitis is a sign of widespread inflammation and that pleural effusion in one or both sides of the chest is associated with a poor outcome (Level 2b). ${ }^{54-56}$ Because ileus found by plain abdominal $x$-rays may be a sign of organ failure, its association with severity should be investigated in further research.

\section{Angiography}

Diagnosis of acute pancreatitis itself does not require angiography. However, in Japan, angiography is sometimes carried out in the acute phase of acute pancreatitis before the performing of continuous regional arterial infusion of protease inhibitors and antibacterial drugs. The angiographic findings may be helpful in diagnosing the severity of the disease. Arterial constriction observed by angiography suggests the presence of splanchnic ischemia of the pancreas and other intraperitoneal organs, and is correlated with severity (Level 2b). 57,58

\section{Severity assessment based on other factors}

\section{Obesity}

Obesity has been found to have a major negative impact on prognosis and the progression of severity of acute parcreatitis in the United States and Europe (Level 1a). ${ }^{59}$ The number of patients with severe pancreatitis with abscess formation and the number of deaths are significantly higher among patients with acute pancreatitis who are obese, particularly those with a body mass index (BMI; body weight $[\mathrm{kg}] /$ height ${ }^{2}\left[\mathrm{~m}^{2}\right]$ ) of $30 \mathrm{~kg} / \mathrm{m}^{2}$ or more (Level 1c-2c). ${ }^{60,61}$ This is explained by local complications caused by obesity (Level 2b) ${ }^{62}$ and the higher probability of respiratory complications from obesity (Level 3b). ${ }^{63}$

\section{Gastric intramucosal pH (pHi)}

The lowering of $\mathrm{pHi}$ is based on the rapid reduction of circulating plasma volume at the onset of acute pancreatitis and accompanying acute circulation failure. $\mathrm{pHi}$ is measured with a tonometer inserted transnasally within 12 to $48 \mathrm{~h}$ after the onset of acute pancreatitis. A study (Level 1c) ${ }^{64}$ of 17 patients with severe pancreatitis revealed a significantly higher mortality rate among those with the lowest $\mathrm{pHi}$ within the first $48 \mathrm{~h}$ after admission, with a pHi of 7.25 as a cutoff value (sensitivity, $100 \%$; specificity, $77 \%$; and positive predictive value, $82 \%$ ). The number of failed organs was higher among patients with lower pHi (Level 2b). ${ }^{65}$ There are significant corre- 
lations of $\mathrm{pHi}$ with the serum levels of various cytokines (Level 2b), ${ }^{66}$ but pHi cannot be used to differentiate between severe and moderate cases (Level 2b). ${ }^{67}$

\section{Molecular markers}

The blood concentrations of interleukin-6 (Level 2b), ${ }^{28}$ interleukin-8 (Level 2b) ${ }^{68}$ soluble tumor necrosis factor-receptor (sTNF-R; Level 2b), ${ }^{68,69}$ granulocyte esterase (Levels 1c-2b), ${ }^{27,70,71}$ cytochrome c (Level 2b), ${ }^{72}$ creatine phosphokinase (CPK; Level 2b) ${ }^{73}$ activation peptide of procarboxypeptidase B (CAPAP), ${ }^{74,75}$ methemalbumin ${ }^{76}$ procalcitonin, ${ }^{77}$ pancreatitis-associated protein (PAP) ${ }^{78}$ ribonuclease, ${ }^{79}$ and endothelin- ${ }^{80}$ all reflect the severity of acute pancreatitis. Urine trypsinogen activation peptide (TAP; Level 2b) ${ }^{74,81}$ ascites TAP (Level 1b), ${ }^{82}$ and urine trypsinogen-2 (Level 1b) ${ }^{83}$ are also reported to reflect the severity of pancreatitis. In view of their high sensitivity and specificity, these markers are expected to be useful in the future for assessing severity and predicting progression to a poor outcome. Elevation of blood amylase and lipase concentrations is an important factor for the diagnosis of acute pancreatitis, but it does not necessarily reflect severity.

\section{Transfer criteria}

CQ6. What are the indications for transferring patients with acute pancreatitis to a specialist unit?

It is desirable to transfer patients with severe acute pancreatitis (JPN score of 2 or more) to a medical institution where monitoring and systemic management are available (Recommendation $\mathbf{A}$ )

Inpatient treatment is very important with acute pancreatitis. The guidelines of the British Society of Gastroenterology (1998) ${ }^{20}$ recommend that multiple acute fluid collections, contrast-enhanced CT findings that indicate pancreatic necrosis of greater than $50 \%$, and organ failure be used as indications for transfer to a specialist medical institution. The Santorini Consensus Conference in $1999^{19}$ recommended that obesity (BMI, $>30 \mathrm{~kg} / \mathrm{m}^{2}$ ), pleural effusion, an APACHE II score of 6 , or more, an APACHE-O score ("O" stands for obesity) of 6 or more (APACHE II score + 1, if BMI is $25-30 \mathrm{~kg}$ / $\mathrm{m}^{2}$; or +2 , if BMI is $>30 \mathrm{~kg} / \mathrm{m}^{2}$ ), or CRP of more than $15 \mathrm{mg} / \mathrm{dl}$ be used as indications of a severe case in which the patient needs referral to a specialist medical institution.

In Japan, the JPN score ${ }^{11}$ is widely used as the criterion for assessing the severity of acute pancreatitis, and it is desirable to transfer patients diagnosed with severe pancreatitis (based on the JPN score) to a medical institution with full-time physicians and surgeons specializ- ing in digestive diseases. Because the mortality rate for patients with a JPN score of 8 or more within the first 24 to $48 \mathrm{~h}$ of onset and an APACHE II score of 13 or more is significantly higher than that in patients with JPN scores of less than 8 and APACHE II scores of less than 13 , such patients should be transferred to a specialist medical institution with physicians specializing in intensive care, endoscopic treatment, radiological intervention, and biliary-pancreatic surgery (advanced medical unit; Level 3b). Because acute pancreatitis diagnosed as moderate may become severe, the progress of the disease should be carefully monitored (with sufficient fluid replacement) to examine for indications for transfer to a specialist medical institution. The decision to transfer patients should be made after taking into consideration the possible effect of a long road trip on the patient's morbidity.

It is desirable to transfer patients with severe acute pancreatitis (JPN score of 2 or more) to a specialist medical institution where they can receive adequate monitoring and systemic management.

\section{References}

1. Mayumi T, Ura H, Arata S, et al. Evidence-baced clinical practice guidelines for acute pancreatitis: proposals. J Hepatobiliary Pancreat Surg 2002;9:413-22.

2. Kish MA. Guide to development of practice guidelines. Clin Infect Dis 2001;32:851-4.

3. Ranson JH, Rifkind KM, Roses DF, et al. Prognostic signs and the role of operative management in acute pancreatitis. Surg Gynecol Obstet 1974;139:69-81.

4. Ranson JH. Etiological and prognostic factors in human acute pancreatitis: a review. Am J Gastroenterol 1982;77:633-8.

5. Imrie CW, Benjamin IS, Ferguson JC, et al. A single-centre double-blind trial of Trasylol therapy in primary acute pancreatitis. Br J Surg 1978;65:337-41.

6. Osborne DH, Imrie CW, Carter DC. Biliary surgery in the same admission for gallstone-associated acute pancreatitis. Br J Surg 1981;68:758-61.

7. Blamey SL, Imrie CW, O'Neill J, et al. Prognostic factors in acute pancreatitis. Gut 1984;25:1340-6.

8. Bank S, Wise L, Gersten M. Risk factors in acute pancreatitis. Am J Gastroenterol 1983;78:637-40.

9. Forell MM. Die konservative Behandlung der Pankreaserkrankungen. Internist (Berl) 1964;5:453-7.

10. Dammann HG, Dopner M, von Wichert P, et al. Die Beurteilung der Fruhprognose der akuten Pankreatitis. Zentralbl Chir 1981; 106:154-60.

11. Ogawa M, Hirota M, Hayakawa T, et al. Development and use of a new staging system for severe acute pancreatitis based on a nationwide survey in Japan. Pancreas 2002;25:325-30.

12. Knaus WA, Draper EA, Wagner DP, et al. APACHE II: a severity of disease classification system. Crit Care Med 1985;13:818-29.

13. Ranson JH, Rifkind KM, Turner JW. Prognostic signs and nonoperative peritoneal lavage in acute pancreatitis. Surg Gynecol Obstet 1976;143:209-19.

14. Leese T, Shaw D. Comparison of three Glasgow multifactor prognostic scoring systems in acute pancreatitis. Br J Surg 1988;75: 460-2.

15. De Bernardinis M, Violi V, Roncoroni L, Boselli AS, et al. Discriminant power and information content of Ranson's prognostic 
signs in acute pancreatitis: a meta-analytic study. Crit Care Med 1999;27:2272-83

16. Larvin M. Assessment of severity and prognosis in acute pancreatitis. Eur J Gastroenterol Hepatol 1997;9:122-30.

17. Wilson C, Heath DI, Imrie CW. Prediction of outcome in acute pancreatitis: a comparative study of APACHE II, clinical assessment and multiple factor scoring systems. Br J Surg 1990;77:12604.

18. Bradley EL III. A clinically based classification system for acute pancreatitis. Summary of the International Symposium on Acute Pancreatitis, Atlanta, GA, September 11 through 13, 1992. Arch Surg 1992;128:586-90.

19. Dervenis C, Johnson CD, Bassi C, et al. Diagnosis, objective assessment of severity, and management of acute pancreatitis. Santorini Consensus Conference. Int J Pancreatol 1999;25:195210.

20. British Society of Gastroenterology: United Kingdom guidelines for the management of acute pancreatitis. Gut 1998;42: S1-13.

21. Otsuki M, Ito T, Koizumi M, Shimosegawa T. Mortality and deterioration factors of acute pancreatitis - the multicenter analysis of death from acute pancreatitis (in Japanese with English abstract). Suizou (J Jpn Panc Soc) 2005;20:17-30.

22. Kitagawa M, Naruse T, Hayakawa T, et al. Prediction of exacerbation of acute pancreatitis (in Japanese with English abstract). Suizou (J Jpn Panc Soc) 2001;16:95-102.

23. Dickson AP, Imrie CW. The incidence and prognosis of body wall ecchymosis in acute pancreatitis. Surg Gynecol Obstet 1984;159:343-7.

24. Bem J, Bradley El III. Subcutaneous manifestations of severe acute pancreatitis. Pancreas 1998;16:551-5.

25. Dervenis C, Johnson CD, Bassi C, et al. Diagnosis, objective assessment of severity, and management of acute pancreatitis. Santorini Consensus Conference. Int J Pancreatol 1999;25:195210.

26. Corfield AP, Cooper MJ, Williamson RC, et al. Prediction of severity in acute pancreatitis: prospective comparison of three prognostic indices. Lancet 1985;II:403-7.

27. Viedma JA, Perez MM, Agullo J, et al. Inflammatory response in the early prediction of severity in human acute pancreatitis. Gut 1994;35:822-7.

28. Pezzilli R, Billi P, Miniero R, et al. Serum interleukin-6, interleukin-8, and beta 2-microglobulin in early assessment of severity of acute pancreatitis. Comparison with serum C-reactive protein. Dig Dis Sci 1995;40:2341-8.

29. Uchikov PA, Sirakova IP, Murdjeva MA, et al. Changes in plasma levels of acute phase proteins in pancreatitis. Folia Med (Plovdiv) 2000;42:23-30.

30. Wilson C, Heads A, Imrie CW. C-reactive protein, antiproteases and complement factors as objective markers of severity in acute pancreatitis. Br J Surg 1989;76:177-81.

31. Vesentini S, Bassi C, Talamini G, et al. Prospective comparison of C-reactive protein level, Ranson score and contrast-enhanced computed tomography in the prediction of septic complications of acute pancreatitis. Br J Surg 1993;80:755-7.

32. Lankisch PG, Petersen M, Gottesleben F. High, not low, amylase and lipase levels indicate severe acute pancreatitis! Z Gastroenterol 1994;32:213-5.

33. Balthazar EJ, Robinson DL, Megibow AJ, et al. Acute pancreatitis: value of CT in establishing prognosis. Radiology 1990; 174:331-6.

34. Brown A, Orav J, Banks PA. Hemoconcentration is an early marker for organ failure and necrotizing pancreatitis. Pancreas 2000;20:367-72.

35. Baillargeon JD, Ramagopal V, Tenner SM, et al. Hemoconcentration as an early risk factor for necrotizing pancreatitis. Am J Gastroenterol 1998;93:2130-4.

36. Matsuda Y, Ogawa M, Nishijima J, et al. Usefulness of determination of serum immunoreactive pancreatic phospholipase $\mathrm{A}_{2}$ con- tent for early identification of severe acute pancreatitis. Hepatogastroenterology 1986;33:214-6.

37. Nevalainen TJ, Gronroos JM, Kortesuo PT. Pancreatic and synovial type phospholipases $A_{2}$ in serum samples from patients with severe acute pancreatitis. Gut 1993;34:1133-6.

38. Hietaranta A, Kemppainen E, Puolakkainen P, et al. Extracellular phospholipases $\mathrm{A}_{2}$ in relation to systemic inflammatory response syndrome (SIRS) and systemic complications in severe acute pancreatitis. Pancreas 1999;18:385-91.

39. Kemppainen E, Sainio V, Haapiainen R, et al. Early localization of necrosis by contrast-enhanced computed tomography can predict outcome in severe acute pancreatitis. Br J Surg 1996;83:9249.

40. Bradley EL III. Prediction of pancreatic necrosis by dynamic pancreatography. Ann Surg 1989;210:495-503.

41. Larvin M, Chalmers AG, McMahon MJ. Dynamic contrastenhanced computed tomography: a precise technique for identifying and localizing pancreatic necrosis. BMJ 1990;300:1425-8.

42. Hwang TL, Chang KY, Ho YP. Contrast-enhanced dynamic computed tomography does not aggravate the clinical severity of patients with severe acute pancreatitis: reevaluation of the effect of intravenous contrast medium on the severity of acute pancreatitis. Arch Surg 2000;135:287-90.

43. Carmona-Sanchez R, Uscanga L, Bezaury-Rivas P, et al. Potential harmful effect of iodinated intravenous contrast medium on the clinical course of mild acute pancreatitis. Arch Surg 2000; 135:1280-4.

44. Casas JD, Diaz R, Valderas G, et al. Prognostic value of CT in the early assessment of patients with acute pancreatitis. AJR Am J Roentgenol 2004;182:569-74.

45. Mortele KJ, Wiesner W, Intriere L, et al. A modified CT severity index for evaluating acute pancreatitis: improved correlation with patient outcome. AJR Am J Roentgenol 2004;183:12615.

46. Matsuno S, Takeda K, Ogawa M, Hirota M. CT staging of the severity of acute pancreatitis: analysis of data collected by Research Committee for Intractable Disease of the Pancreas in Japan (in Japanese with English abstract). Suizou (J Jpn Panc Soc) 2002;17:93-9.

47. London NJ, Leese T, Lavelle JM, et al. Rapid-bolus contrastenhanced dynamic computed tomography in acute pancreatitis: a prospective study. Br J Surg 1991;78:1452-6.

48. Rotman N, Chevret S, Pezet D, et al. Prognostic value of early computed tomographic scans in severe acute pancreatitis. J Am Coll Surg 1994;179:538-44.

49. Clavien PA, Hauser H, Meyer P, et al. Value of contrastenhanced computerized tomography in the early diagnosis and prognosis of acute pancreatitis. Am J Surg 1988;155:45766.

50. Hirota M, Kimura Y, Ishiko T, et al. Visualization of the heterogeneous internal structure of so-called pancreatic necrosis by magnetic resonance imaging. Pancreas 2002;25:63-7.

51. Ward J, Chalmers AG, Guthrie AJ, et al. T2-weighted and dynamic enhanced MRI in acute pancreatitis: comparison with contrast enhanced CT. Clin Radiol 1997;52:109-14.

52. Lecesne R, Taourel P, Bret PM, et al. Acute pancreatitis: interobserver agreement and correlation of $\mathrm{CT}$ and $\mathrm{MR}$ cholangiopancreatography with outcome. Radiology 1999;211: 727-35.

53. Arvanitakis M, Delhaye M, De Maetelaere V, et al. Computed tomography and magnetic resonance imaging in the assessment of acute pancreatitis. Gastroenterology 2004;126:715-23.

54. Talamini G, Bassi C, Falconi M, et al. Risk of death from acute pancreatitis. Role of early, simple "routine" data. Int J Pancreatol 1996;19:15-24.

55. Maringhini A, Ciambra M, Patti R, et al. Ascites, pleural, and pericardial effusions in acute pancreatitis. A prospective study of incidence, natural history, and prognostic role. Dig Dis Sci 1996;41:848-52. 
56. Lankisch PG, Droge M, Becher R. Pulmonary infiltrations. Sign of severe acute pancreatitis. Int J Pancreatol 1966;19:113-5.

57. Inoue K, Hirota M, Beppu T, et al. Angiographic features in acute pancreatitis: the severity of abdominal vessel ischemic change reflects the severity of acute pancreatitis. J Pancreas 2003;4:20713.

58. Takeda K, Mikami Y, Fukuyama S, Egawa S, Sunamura M, Ishibashi T, et al. Pancreatic ischemia associated with vasospasm in the early phase of human acute necrotizing pancreatitis. Pancreas 2005;30:40-9.

59. Martinez J, Sanchez-Paya J, Palazon JM, Suazo-Barahona J, Robles-Diaz G, Perez-Mateo M. Is obesity a risk factor in acute pancreatitis? A meta-analysis. Pancreatology 2004;4:42-8.

60. Funnell IC, Bornman PC, Weakley SP, et al. Obesity: an important prognostic factor in acute pancreatitis. Br J Surg 1993;80:4846.

61. Martinez J, Sanchez-Paya J, Palazon JM, et al. Obesity: a prognostic factor of severity in acute pancreatitis. Pancreas 1999;19:1520.

62. Tsai CJ. Is obesity a significant prognostic factor in acute pancreatitis? Dig Dis Sci 1998;43:2251-4.

63. Porter KA, Banks PA. Obesity as a predictor of severity in acute pancreatitis. Int J Pancreatol 1991;10:247-52.

64. Bonham MJ, AbuZidan F, Simovic MO, et al. Gastric intramucosal $\mathrm{pH}$ predicts death in severe acute pancreatitis. Br J Surg 1997;84:1670-4.

65. Hynninen M, Valtonen M, Markkanen $\mathrm{H}$, et al. Intramucosal $\mathrm{pH}$ and endotoxin and cytokine release in severe acute pancreatitis. Shock 2000;13:79-82.

66. Soong CV, Lewis HG, Halliday MI, et al. Intramucosal acidosis and the inflammatory response in acute pancreatitis. Am J Gastroenterol 1999;94:2423-9.

67. Juvonen PO, Alhava EM, Takala JA. Gastric tonometry in assessing splanchnic tissue perfusion in acute pancreatitis. Scand J Gastroenterol 2000;35:318-21.

68. De Beaux AC, Goldie AS, Ross JA, et al. Serum concentrations of inflammatory mediators related to organ failure in patients with acute pancreatitis. Br J Surg 1996;83:349-53.

69. Hirota M, Nozawa F, Okabe A, et al. Relationship between plasma cytokine concentration and multiple organ failure in patients with acute pancreatitis. Pancreas 2000;21:141-6.

70. Gross V, Scholmerich J, Leser HG, et al. Granulocyte elastase in assessment of severity of acute pancreatitis. Comparison with acute-phase proteins C-reactive protein, alpha 1-antitrypsin, and protease inhibitor alpha 2-macroglobulin. Dig Dis Sci 1990;35:97105.

71. Uhl W, Buchler M, Malfertheiner P, et al. PMN-elastase in comparison with CRP, antiproteases, and LDH as indicators of necrosis in human acute pancreatitis. Pancreas 1991;6:253-9.

72. Adachi N, Hirota M, Hamaguchi M, et al. Serum cytochrome c level as a prognostic indicator in patients with systemic inflammatory response syndrome. Clin Chim Acta 2004;342:127-36.

73. Hirota M, Inoue K, Kimura Y, et al. Non-occlusive mesenteric ischemia and its associated intestinal gangrene in acute pancreatitis. Pancreatology 2003;3:316-22.

74. Saez J, Martinez J, Trigo C, et al. A comparative study of the activation peptide of carboxypeptidase B and trypsinogen as early predictors of the severity of acute pancreatitis. Pancreas 2004; 29:e9-e14.

75. Appelros S, Thim L, Borgstrom A. Activation peptide of carboxypeptidase $B$ in serum and urine in acute pancreatitis. Gut 1998;42:97-102.

76. Lankisch PG, Schirren CA, Otto J. Methemalbumin in acute pancreatitis: an evaluation of its prognostic value and comparison with multiple prognostic parameters. Am J Gastroenterol 1989; 84:1391-5.

77. Riche FC, Cholley BP, Laisne MJ, et al. Inflammatory cytokines, $\mathrm{C}$-reactive protein, and procalcitonin as early predictors of necrosis infection in acute necrotizing pancreatitis. Surgery 2003; 133:257-62.

78. Iovanna JL, Kein V, Nordback I, et al. Serum levels of pancreatitis-associated protein as indicators of the course of acute pancreatitis. Gastroenterology 1994;106:728-34.

79. Warshow AL, Lee KH. Serum ribonuclease elevation and pancreatic necrosis in acute pancreatitis. Surgery 1979;86:227-34.

80. Inoue $\mathrm{K}$, Hirota $\mathrm{M}$, Kimura $\mathrm{Y}$, et al. Further evidence for endothelin as an important mediator of pancreatic and intestinal ischemia in severe acute pancreatitis. Pancreas 2003;26:218-23.

81. Neoptolemos JP, Kemppainen EA, Mayer JM, et al. Early prediction of severity in acute pancreatitis by urinary trypsinogen activation peptide: a multicentre study. Lancet 2000;355:1955-60.

82. Heath DI, Wilson C, Gudgeon AM, et al. Trypsinogen activation peptides (TAP) concentrations in the peritoneal fluid of patients with acute pancreatitis and their relation to the presence of histologically confirmed pancreatic necrosis. Gut 1994;35:1311-5.

83. Lempinen M, Stenman UH, Finne P, et al. Trypsinogen-2 and trypsinogen activation peptide (TAP) in urine of patients with acute pancreatitis. J Surg Res 2003;111:267-73. 